\section{Blastic plasmacytoid dendritic cell neoplasm: genomics mark epigenetic dysregulation as a primary therapeutic target}

\author{
Maria Rosaria Sapienza,,$^{1^{\star}}$ Francesco Abate,,$^{2,3^{\star}}$ Federica Melle, ${ }^{4}$ \\ Stefania Orecchioni, ${ }^{5}$ Fabio Fuligni, ${ }^{6}$ Maryam Etebari, ${ }^{1}$ Valentina Tabanelli, ${ }^{4}$ \\ Maria Antonella Laginestra, ${ }^{1}$ Alessandro Pileri, ${ }^{7,8}$ Giovanna Motta, ${ }^{4}$ \\ Maura Rossi, ${ }^{1}$ Claudio Agostinelli, ${ }^{1}$ Elena Sabattini, ${ }^{1}$ Nicola Pimpinelli, ${ }^{8}$ \\ Mauro Truni, ${ }^{9}$ Brunangelo Falini, ${ }^{10}$ Lorenzo Cerroni, ${ }^{11}$ Giovanna Talarico, ${ }^{5}$ \\ Rossana Piccioni, ${ }^{12}$ Stefano Amente, ${ }^{13}$ Valentina Indio, ${ }^{14}$ \\ Giuseppe Tarantino, ${ }^{14}$ Francesco Brundu, ${ }^{2}$ Marco Paulli, ${ }^{15}$ Emilio Berti, ${ }^{16}$ \\ Fabio Facchetti, ${ }^{17}$ Gaetano Ivan Dellino, ${ }^{12,18}$ Francesco Bertolini, ${ }^{5}$ \\ Claudio Tripodo, ${ }^{1{ }^{*}}$ Raul Rabadan ${ }^{2,3^{*}}$ and Stefano A. Pileri ${ }^{4 \star *}$
}

${ }^{1}$ Hematopathology Unit, Department of Experimental, Diagnostic, and Specialty Medicine, S. Orsola-Malpighi Hospital, University of Bologna, Italy; ${ }^{2}$ Department of Systems Biology, Columbia University College of Physicians and Surgeons, New York, NY, USA; ${ }^{3}$ Department of Biomedical Informatics, Columbia University College of Physicians and Surgeons, New York, NY, USA; ' ${ }^{2}$ Division of Haematopathology, IEO European Institute of Oncology IRCCS, Milan, Italy; ${ }^{5}$ Laboratory of Hematology-Oncology, IEO European Institute of Oncology IRCCS, Milan, Italy; ${ }^{6}$ Department of Genetics and Genome Biology, The Hospital for Sick Children, Toronto, ON, Canada: ${ }^{7}$ Dermatology Unit, Department of Experimental, Diagnostic and Specialty Medicine, University of Bologna, Italy; ${ }^{8}$ Division of Dermatology, Department of Surgery and Translational Medicine, University of Florence, Italy; ${ }^{9}$ Pathological Anatomy Histology \& Cytogenetics, Niguarda Cancer Center, Niguarda-Ca' Granda Hospital, Milan, Italy; ${ }^{10}$ Institute of Hematology and Center for Hemato-Oncology Research (CREO), University and Hospital of Perugia, Italy; ${ }^{11}$ Universitätsklinik für Dermatologie und Venerologie, $\mathrm{LKH}$ Universitatsklinikum Graz, Austria; ${ }^{12}$ Department of Experimental Oncology, European Institute of Oncology, Milan, Italy; ${ }^{13}$ Department of Molecular Medicine and Medical Biotechnologies, University of Naples 'Federico II', Italy; ${ }^{14}$ "Giorgio Prodi" Cancer Research Center, University of Bologna, Italy; ${ }^{15}$ Unit of Anatomic Pathology, Department of Molecular Medicine, University of Pavia and Fondazione IRCCS San Matteo Policlinic, Pavia, Italy; ${ }^{16}$ Department of Dermatology, Fondazione IRCCS Ca' Granda - Ospedale Maggiore Policlinic and Milan University, Milan, Italy; ${ }^{17}$ Pathology Section, Department of Molecular and Translational Medicine, University of Brescia, Italy; ${ }^{18}$ Department of Oncology and Hemato-Oncology, University of Milan, Italy and ${ }^{19}$ Tumor Immunology Unit, Department of Health Science, Human Pathology Section, University of Palermo School of Medicine, Italy

${ }^{\star} M R S, F A, C T, R R$ and SAP contributed equally to this work. ${ }^{\ddagger}$ Alma Mater Professor, Bologna University

\section{ABSTRACT}

$\mathrm{B}$ lastic plasmacytoid dendritic cell neoplasm (BPDCN) is a rare and aggressive hematologic malignancy for which there is still no effective therapy. In order to identify genetic alterations useful for a new treatment design, we used whole-exome sequencing to analyze 14 BPDCN patients and the patient-derived CAL-1 cell line. The functional enrichment analysis of mutational data reported the epigenetic regulatory program to be the most significantly undermined $(P<0.0001)$. In particular, twenty-five epigenetic modifiers were found mutated (e.g. ASXL1, TET2, SUZ12, ARID1A, PHF2, CHD8); ASXL1 was the most frequently affected $(28.6 \%$ of cases). To evaluate the impact of the identified epigenetic mutations at the gene-expression and Histone H3 lysine 27 trimethylation/acetylation levels, we performed additional RNA and pathology tissue-chromatin immunoprecipitation sequencing experiments. The patients displayed enrichment in gene signatures regulated by methylation and modifiable by decitabine administration, shared common H3K27-acetylated regions, and had a set of cell-cycle genes aberrantly up-regulated and marked by promoter acetylation. Collectively, the integration of sequencing data showed the potential of a therapy based on epigenetic agents. Through the adoption of a preclinical BPDCN mouse model, established by CAL-1 cell line xenografting, we demonstrated the efficacy of the combination of the epigenetic drugs 5 'azacytidine and decitabine in controlling disease progression in vivo.
Haematologica 2019

Volume 104(4):729-737

\section{Correspondence:}

MARIA ROSARIA SAPIENZA

mariarosaria.sapienza@gmail.com

Received: July 16, 2018.

Accepted: October 30, 2018.

Pre-published: October 31, 2018.

doi:10.3324/haematol.2018.202093

Check the online version for the most updated information on this article, online supplements, and information on authorship \& disclosures: www.haematologica.org/content/104/4/729

\section{(C)2019 Ferrata Storti Foundation}

Material published in Haematologica is covered by copyright. All rights are reserved to the Ferrata Storti Foundation. Use of published material is allowed under the following terms and conditions:

https://creativecommons.org/licenses/by-nc/4.0/legalcode. Copies of published material are allowed for personal or internal use. Sharing published material for non-commercial purposes is subject to the following conditions:

https://creativecommons. org/licenses/by-nc/4.0/legalcode, sect. 3. Reproducing and sharing published material for commercial purposes is not allowed without permission in writing from the publisher. 


\section{Introduction}

Blastic plasmacytoid dendritic cell neoplasm (BPDCN) is a rare malignancy derived from precursors of plasmacytoid dendritic cells. ${ }^{1-4}$ It has no clear racial or ethnic predisposition and more often affects elderly males (male/female ratio 3.3:1; mean/median age at diagnosis: 61-67 years). BPDCN patients usually respond to firstline chemotherapy, but despite this they almost invariably relapse and display a dismal prognosis with a median overall survival (OS) ranging from 10 to 19 months. ${ }^{2}$ No standardized therapeutic approach has so far been established for BPDCN, even if hematopoietic stem cell transplantation has been shown to achieve remission in selected patients. ${ }^{5 \cdot 6}$ Therefore, the development of effective treatments still represents an unmet need.' The pathobiology of BPDCN is poorly understood and the number of reports exploring its molecular features is still limited. ${ }^{8-21}$ Recent advances in the understanding of the BPDCN molecular landscape have paved the way for novel treatment approaches based on the inhibition of the BCL2 protein, ${ }^{22}$ the activation of the cholesterol efflux ${ }^{23}$ the repression of the Bromodomain-containing protein 4 (BRD4), ${ }^{24}$ and binding to the interleukin-3 receptor (IL3R). ${ }^{25}$ All these potential therapeutic options (which are worthy of further evaluation) have mainly emerged from the analysis of the BPDCN transcriptome or from its antigenic repertoire. The genomic landscape of BPDCN has not been well investigated, and only a few studies have explored the mutational events occurring in $\mathrm{BPDCN}$, mainly through targeted sequencing approaches. ${ }^{14,16,19,20}$

Unfortunately, these have not offered any novel prospects of treatment options.

In this study, we performed whole-exome sequencing (WES) of 14 BPDCN samples and of the BPDCN-derived CAL-1 cell line to look for specific BPDCN genetic vulnerabilities that may support the design of new therapeutic strategies. The WES mutational findings were complemented by copy number variant (CNV) analysis, RNA and pathology tissue-chromatin immunoprecipitation (PATChIP) sequencing results. The integration of data allowed us to identify a successful combinatorial therapy based on epigenetic drugs able to control disease progression in a rapidly progressive BPDCN xenograft model.

\section{Methods}

\section{Blastic plasmacytoid dendritic cell neoplasm samples}

We collected 14 BPDCN cryopreserved cutaneous biopsies at diagnosis, 9 matched saliva samples and the BPDCN patient-derived cell line, CAL-1. The pathological cases were evaluated as previously described ${ }^{17}$ and diagnosed by experienced hematopathologists (CA, EB, FF, LC, MP, ES, CT, MT, and SAP) according to World Health Organization diagnostic criteria. ${ }^{2}$ Informed consent was obtained from each patient in accordance with the Ethical Review Board of the Department of Experimental, Diagnostic, and Specialty Medicine of the University of Bologna, Italy, and the Declaration of Helsinki. DNA was extracted as reported in the Online Supplementary Appendix. The main clinical, immunohistochemical and cytogenetic features of the BPDCN patients are shown in Online Supplementary Tables S1 and S2.

\section{Whole-exome sequencing analysis}

We performed paired-end sequencing of matched tumor/normal DNA samples (9 cases), tumor only DNA samples (5 cases), and the CAL-1 cell line (Online Supplementary Table S3) using the TruSeq Exome Kit and Nextera Rapid Capture Exome kit (Illumina). Further details are available in the Online Supplementary Appendix.

\section{Sanger sequencing}

We used Sanger sequencing to analyze two candidate nonsense somatic mutations of SUZ12 and ASXL1 occurring in 2 patients, respectively, as described in the Online Supplementary Appendix.

\section{Targeted sequencing}

We performed MiSeq targeted sequencing (Illumina) of the 14 BPDCN tumor patients, 7 normal matched saliva samples and the CAL-1 cell line, already analyzed by WES. More bioinformatics details are provided in the Online Supplementary Appendix and Online Supplementary Tables S4 and $S 5$.

\section{RNA sequencing analysis}

Five BPDCN cases studied by WES and targeted sequencing had sufficient material for RNA extraction and sequencing; these samples represented the RNA sequencing (RNAseq) discovery set. We also collected an additional 4 BPDCN cryopreserved cutaneous biopsies, sufficient only for RNA sequencing experiments, used as an RNA-seq extension set. RNA of 4 normal plasmacytoid dendritic cell (pDCs) samples was purchased from AllCells (Alameda, CA, US) and used for comparison. For details, see Online Supplementary Table S6 and the Online Supplementary Appendix.

\section{Pathology tissue-chromatin immunoprecipitation sequencing}

The BPDCN_25 and BPDCN_37 patients were provided with one skin biopsy: half was cryopreserved and used for WES, targeted and RNA sequencing analysis, and the other half was fixed in formalin, included in paraffin and used for pathology tissue-chromatin immunoprecipitation (PATChIP) sequencing analysis. PAT-ChIP experiments were performed as in Fanelli et al. ${ }^{26}$ Further details are available in the Online Supplementary Appendix.

\section{CAL-1 cell line}

CAL-1, a BPDCN cell line ${ }^{27}$ was cultured as reported previously. ${ }^{13}$ The CAL-1 gene expression profile of a previous study was used ${ }^{17}$ (http://www.ncbi.nlm.nih.gov/geolquery/ acc.cgi? acc=GSE62014).

\section{Mouse model and in vivo treatments}

Experiments were carried out on 6-8-week old nonobese diabetic severe combined immunodeficient NOD/SCID interleukin-2 receptor g (IL-2Rg)-null (NSG) mice, as previously reported. ${ }^{13}$ All animal experiments were carried out in accordance with the Italian laws in force (Legislative Decree 26/14 and subsequent amendments) and institutional guidelines. All in vivo studies were ratified by the Italian Ministry of Health. For induction of BPDCN in mice, 5000 CAL-1 cells were injected intravenously (i.v.) through the lateral tail vein in non-irradiated mice. Engrafted mice were then treated with bortezomib, 5'-azacytidine, decitabine and romidepsin, as detailed in the Online Supplementary Appendix. 


\section{Results}

Whole-exome sequencing reveals the epigenetic program dysregulation as the main theme of the blastic plasmacytoid dendritic cell neoplasm mutational landscape

We collected 14 BPDCN cases with a mean age of 56 years at diagnosis (range 9-89 years), a male-to-female ratio of 10:4, and the classical BPDCN presentation (Online Supplementary Tables S1 and S2). ${ }^{1}$ The enrolled patients underwent different treatment regimens and $78.5 \%$ (11 out of 14) died of the disease 6.3-76 months after the diagnosis or were lost at follow up. Most patients who underwent autologous and/or allogeneic hematopoietic stem cell transplantation experienced a prolonged survival.

We performed WES on 14 BPDCN cases, and on the
A

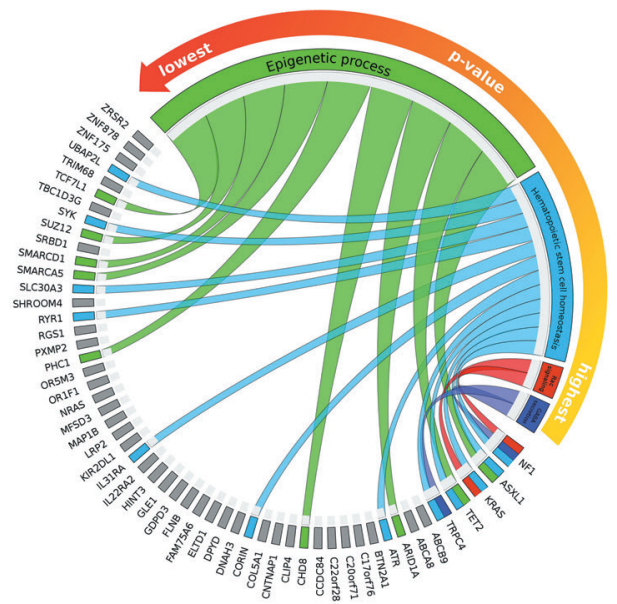

B

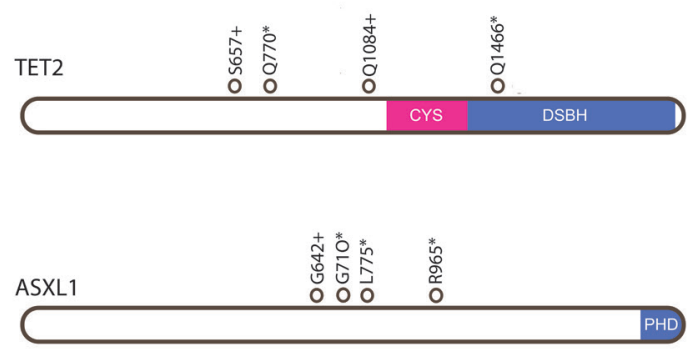

C

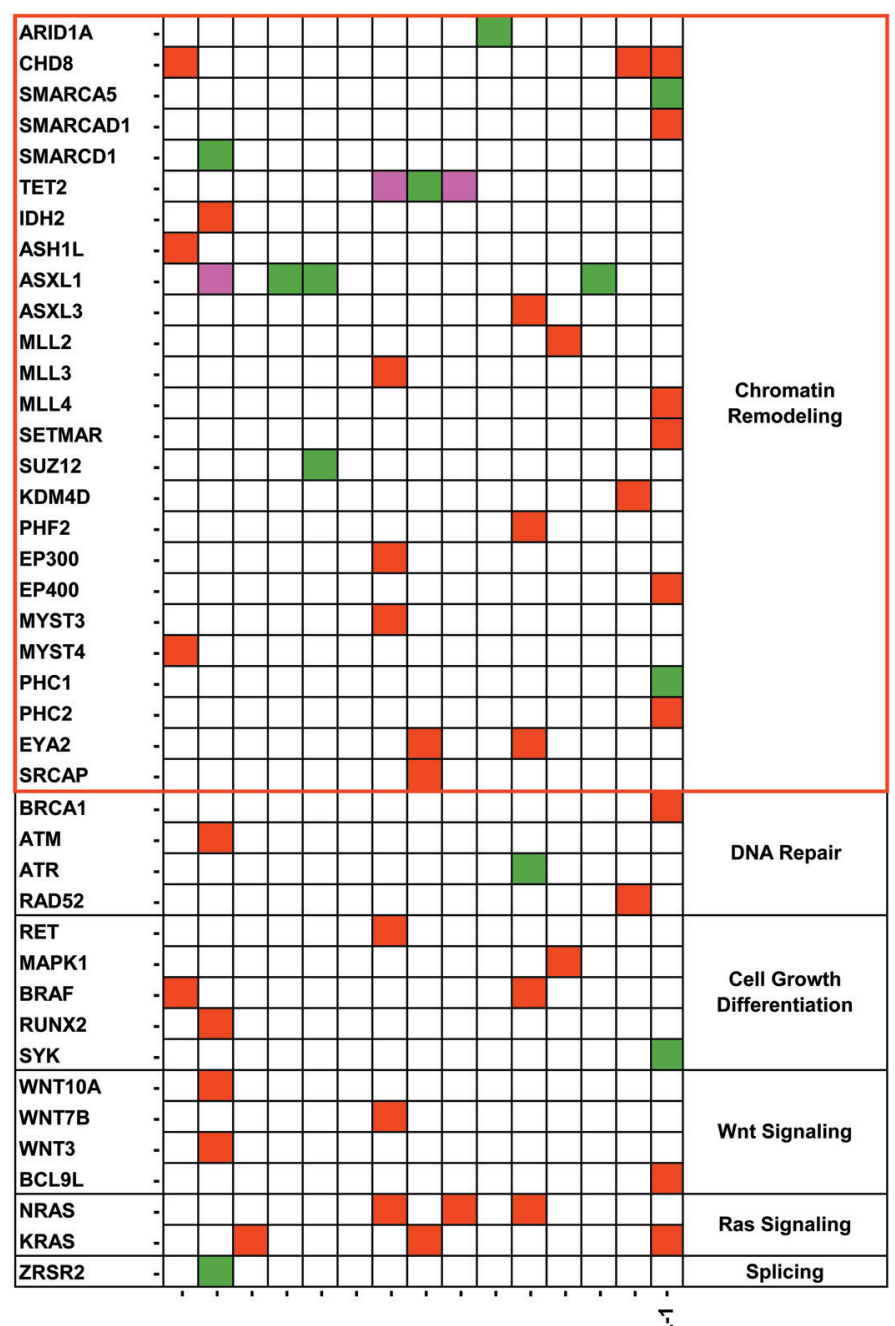

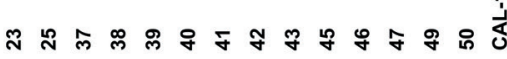

MISSENSE

NONSENSE

FRAMESHIFT

Figure 1. The genomic characterization of blastic plasmacytoid dendritic cell neoplasm (BPDCN). (A) Circos plot graphical representation of the functional analysis performed on 54 genes recurrently mutated and/or affected by nonsense and frameshift single nucleotide variants (SNVs) in BPDCN whole-exome sequencing (WES) samples. The four biologica processes most significantly enriched are reported in the counterclockwise order from the highest to the lowest $P$-value: the gamma-aminobutyric acid (GABA) secretion (in violet), the Rac signaling (in red), the hematopoietic stem cell homeostasis (in light blue) and the epigenetic process (in green). The genes are colored according to their belonging to one or more of the biological processes represented. Genes not involved are in gray. (B) Overview of the TET2 and ASXL1 mutations identified. Structure of ASXL1 protein with C-terminal plant homeodomain catalytic region and structure of TET2 protein showing the catalytic core region: the cysteinerich (Cys) and double-stranded $\beta$-helix (DSBH) domains. Empty circles: somatic SNVs. + : frameshift SNV; * nonsense SNV. (C) Heatmap representation of SNVs in BPDCN WES samples and its distribution among selected pathways commonly mutated in myeloid disorders. The SNVs, the affected genes and the related pathways are reported in rows, while, the BPDCN samples are in columns. 
A

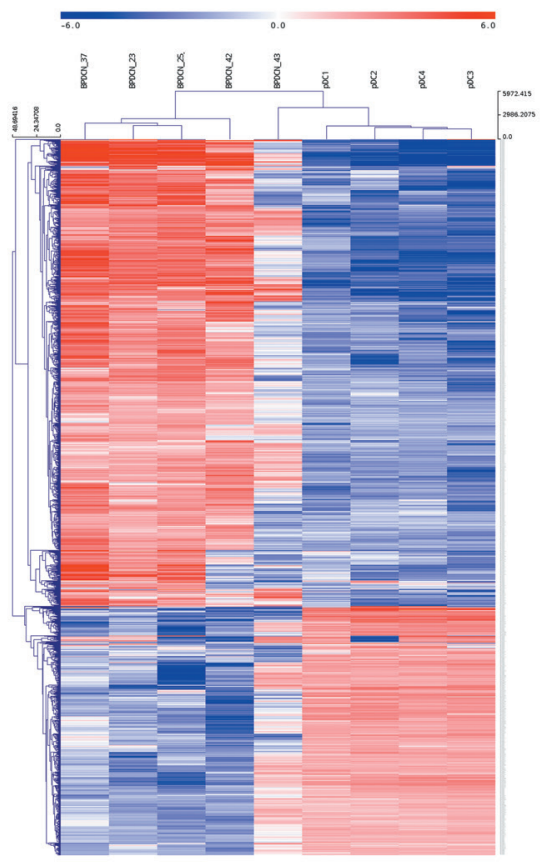

B

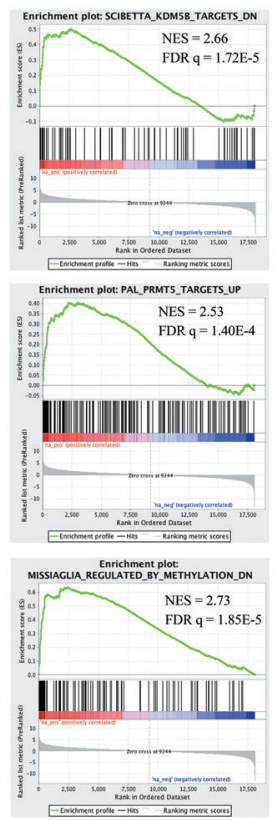

C

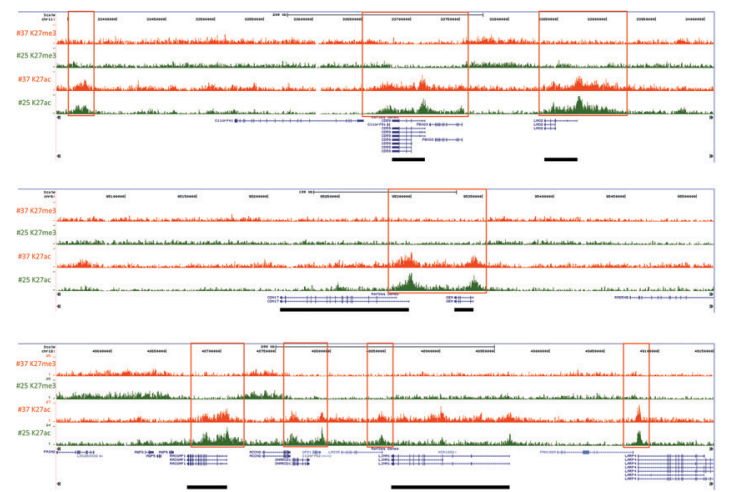

D

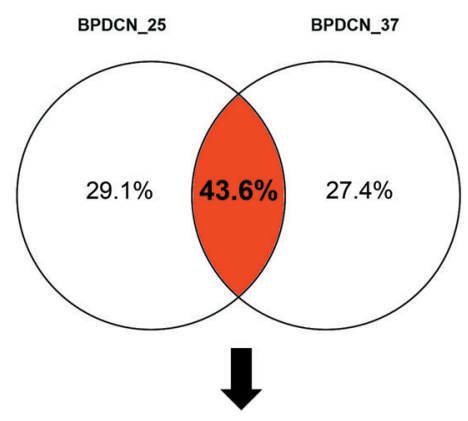

Common H3K27Ac promoters
E

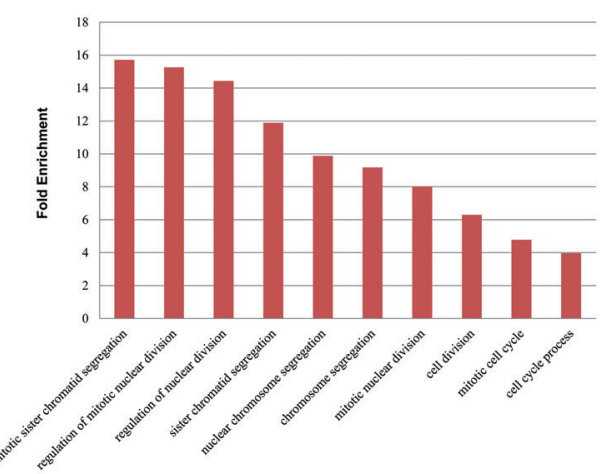

Figure 2. The transcriptome and H3K27 trymethylation/acetylation profiling of blastic plasmacytoid dendritic cell neoplasm (BPDCN). (A) Unsupervised hierarchical clustering performed on 5 BPDCN samples and 4 plasmacytoid dendritic cell (pDCs) samples according to the expression level of the RNA sequencing data. In the heat-map each row represents a gene and each column a sample. The color scale exemplifies the relative expression level of a gene across all samples: (red) represented genes with an expression level above the mean; (blue) the genes with an expression level lower than the mean. Tumors (BPDCNs) and controls ( $p D C s$ ) cluster in two distinct groups. (B) Gene Set Enrichment Analysis (GSEA) plot illustrating the enrichment of the KDM5B and PRMT5 gene signatures in BPDCN patients reported in literature ${ }^{3436}$ as well as the enrichment of a set of genes, described by Missiaglia et al. ${ }^{37}$ as responsive to hypomethylating treatment, namely decitabine. Normalized enrichment score (NES) $\geq 2$; false discovery rate (FDR) q-value false discovery rate $\leq 0.0001$. (C) Visualization of anti-H3K27ac and anti-H3K27me3 normalized pathology tissue-chromatin immunoprecipitation (PAT-ChIP) sequencing profiles in the UCSC Genome Browser showing genomic regions from patient BPDCN_25 and BPDCN_37. (Red boxes) Exemplificative regions displaying a similar level of anti-H3K27ac in both patients. (Black solid rectangles) Genes in correspondence of the anti-H3K27ac peaks. (D) The cases BPDCN_25 and BPDCN_37 share common H3k27ac regions. (E) Histogram representation of the top 10 significant biological processes emerged by Gene Ontology (GO) analysis of 86 up-regulated genes marked by H3K27ac in their promoters. GO categories are shown in $\mathrm{x}$-axis and the fold enrichment values of observed versus expected genes are reported in the $y$-axis (FDR q-value $<0.001$ ). 
A
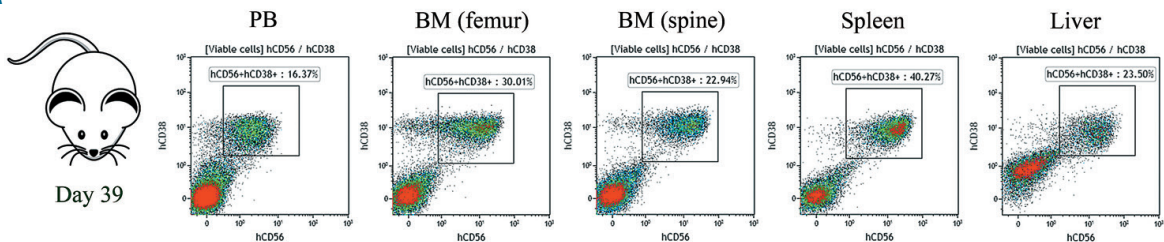

B

BM (H\&E)

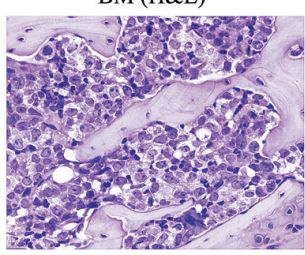

Spleen (H\&E)

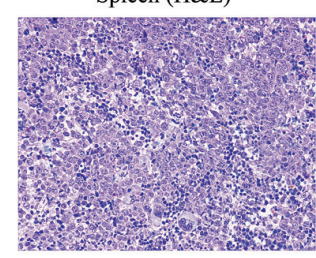

BM (CD303/BDCA2)

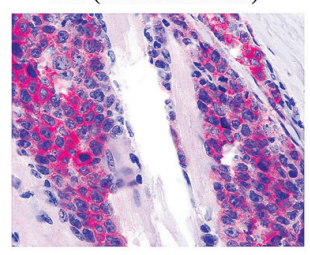

C

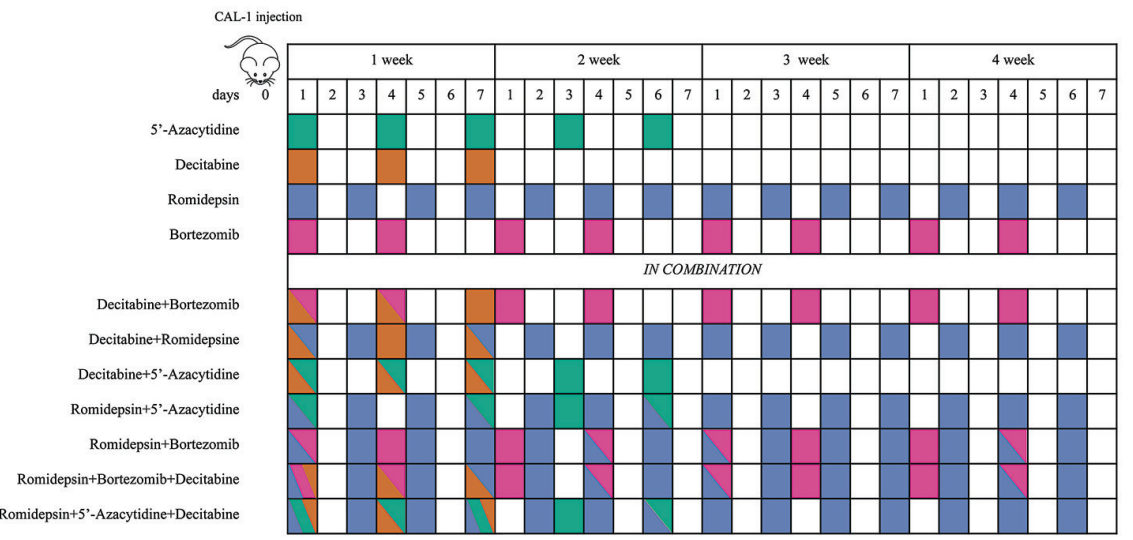

D
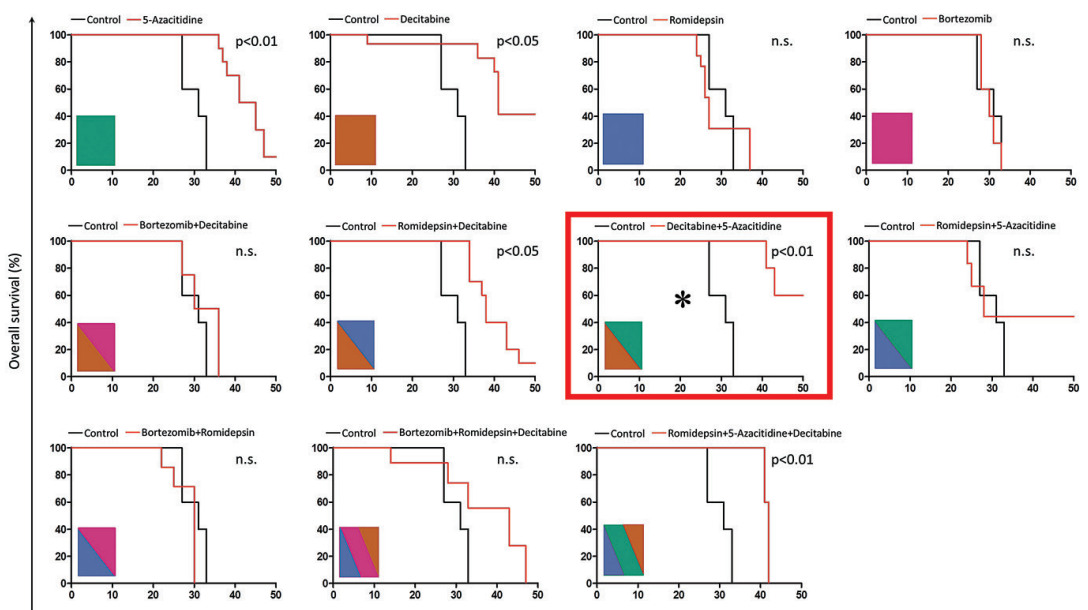

E

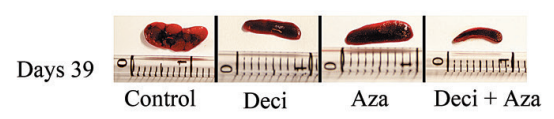

Figure 3. The efficacy of epigenetic agents in a preclinical blastic plasmacytoid dendritic cell neoplasm (BPDCN) mouse model. (A) Pharmacodynamic assessment of the percentage of human $\mathrm{CD} 56^{+} \mathrm{CD} 38^{+}$cells in the peripheral blood (PB), bone marrow (BM) of the femur and spine, spleen, and liver of a representative vehicletreated BPDCN mouse model, 39 days after CAL-1 injection. The cytofluorometric assays shows the tumor dissemination in all the tissues analyzed. (B) Hematoxylin \& eosin (H\&E) staining of BM and spleen samples collected in a representative vehicle-treated NSG mouse 39 days after CAL-1 injection (H\&E; $x 400 ;$ Olympus DP2 $\mathrm{SAL}$ ). The histological assay shows a marked dissemination of blast elements. The immunohistochemistry detection of the CD303 (BDCA-2) antigen, in the murine $\mathrm{BM}$, indicates the presence of specific BPDCN blasts cells (Immmuno-alkaline phosphatase; Gill's hematoxylin nuclear counterstaining; x400; Olympus DP2-SAL). These results further confirmed the effective engraftment of CAL-1 cell line. (C) Graphical representation of the treatment schedules observed in a BPDCN mouse model. Each treatment is represented by a single color or by a combination of colors and was administered for four weeks as follows: 5 '-azacytidine 5 mg/kg 5 doses at 2-day intervals (green), decitabine $2.5 \mathrm{mg} / \mathrm{kg} 3$ doses at 2-day intervals (light brown), romidepsin $0.5 \mathrm{mg} / \mathrm{kg}$ every day (violet), bortezomib $0.5 \mathrm{mg} / \mathrm{kg}$ two times weekly (fuchsia). The same doses were also administered in various combinations. (D) Kaplan-Meier curves comparing overall survival of BPDCN mice models respectively treated with vehicle or the above reported treatments. Each treatment is summarized by a box colored as described above. *Indicates that the combination of decitabine and 5'-azacytidine was the most effective in prolonging mice survival. Curves were compared by log-rank test, $\mathrm{n}=5$ mice/treatment arm. (E) Pharmacodynamic assessment of spleen size in 4 representative NSG mice CAL-1 injected after 39 days of treatment with vehicle (mouse Control), Decitabine (mouse Deci), 5'-azacytidine (mouse Aza), and 5'-azacytidine plus decitabine (mouse Deci+Aza) according to the dosing schedule reported above. ns: not significant. 
BPDCN patient-derived CAL-1 cell line and identified 1302 non-synonymous single nucleotide variants (SNVs) represented predominantly by missense SNVs $(n=1251)$, nonsense SNVs $(n=47)$, and frameshift insertions/deletions ( $\mathrm{n}=7)$. To verify the robustness of the WES analysis, 2 randomly-selected variants occurring in the ASXL1 and SUZ12 genes, respectively, were validated by Sanger sequencing (Online Supplementary Figure S1). To extend the validation to a higher number of samples and mutations, a targeted sequencing approach was adopted: 21 SNVs were interrogated by MiSeq Illumina technology in the same BPDCN cases analyzed by WES and a concordance of $95.2 \%$ (20 out of $21 \mathrm{SNVs}$ ) was achieved, underlining a high consistency of data (Online Supplementary Table S7). The 1302 non-synonymous SNVs detected by WES affected 1166 genes, all but 7 known to be related with pathological conditions and reported as mutated in the Catalogue of Somatic Mutations in Cancer (COSMIC_v66).

To identify the biological processes that were most altered by the mutational events, we performed a functional enrichment analysis of the 9 genes that were recurrently mutated ( $\geq 3$ samples) and the 45 genes impacted by deleterious (nonsense or frameshift) SNVs. Among the top 10 significantly enriched biological processes, the epigenetic program was the most represented $(P=0.0001)$, followed by hematopoietic stem cell homeostasis, Rac signaling and gamma-aminobutyric acid (GABA) secretion (Figure 1A and Online Supplementary Table S8). The ASXL1 gene proved to be the most frequently mutated ( $28 \%, 4$ out of 14 samples), followed by TET2 (21\%, 3 out of 14 samples); both genes displayed mainly nonsense or frameshift SNVs located within or upstream of the catalytic domain of the proteins, potentially leading to their functional disruption (Figure $1 \mathrm{~B}$ and Online Supplementary Table S9). We consulted the Gene Ontology database ${ }^{28}$ to identify among the 1166 BPDCN mutated genes those implicated in the epigenetic regulation. We found 25 mutated epigenetic modifier genes controlling chromatin accessibility (ARID1a, CHD8, SMARCA1), DNA methylation (TET2, IDH2), or histone post-transcriptional modifications [methylation (ASXL1, SUZ12, MLL family), demethylation (KDM4D), acetylation (EP300, EP400), ubiquitination (PHC1, PHC2), dephosphorylation (EYA2), and exchange (SRCAP)]. Of note, 12 out of 14 BPDCN samples $(86 \%)$ harbored at least one of the 25 epigenetic regulator genes mutated, and specifically 8 out of 14 patients $(57.14 \%)$ presented a deleterious lesion (nonsense/frameshift SNV) (Online Supplementary Table S10). Many SNVs clustered in the histone methylation pathway, specifically in genes belonging to the Polycomb-repressive complex 2 (ASXL1, ASXL3, SUZ12) and in histone methyltransferases (ASHL1, SETMAR, MLL), possibly compromising the integrity of the methylation program. Besides genetic lesions targeting epigenetic regulators, we also detected mutations potentially affecting molecular programs commonly deregulated in myeloid malignancies, such as RAS signaling $^{29}$ (hot-spot SNVs on KRAS or NRAS, alternatively), DNA repair/cycle progression ${ }^{30}$ (SNVs on BRCA1, $A T M, A T R$, and RAD52), Wnt-signaling ${ }^{31}$ (SNVs on WNT3, WNT7B, WNT10 and BCL9L), cell growth ${ }^{32}$ (SNVs on RUNX2, MAPK1), and splicing machinery ${ }^{33}$ (an SNV on ZRSR2) (Figure 1C).

Whole-exome sequencing data were also used for cytogenetic CNV analysis, which highlighted extensive losses along the chromosome 9 and the associated deletion of the tumor suppressor CDKN2A gene in 8 out of 14 BPDCN samples (57\%) (Online Supplementary Figure S2), as already reported in the literature. ${ }^{12,15,20}$ In addition, $\mathrm{CNV}$ analysis showed that deletions affected six of the nine genes recurrently mutated; deletions were always mutually exclusive with mutations (Online Supplementary Figure S3). However, no significant correlation was found between genetic lesions and the clinical data.

\section{Blastic plasmacytoid dendritic cell neoplasm tran- scriptome profiling confirms the dysregulation of epi- genetic programs}

Genetic lesions in key epigenetic modifier genes and in related regulatory networks can induce profound perturbations in the transcriptional homeostasis of the cell. To further substantiate the impact of mutations affecting the chromatin remodeling pathway in BPDCN, we performed RNA sequencing of 5 BPDCNs, considered as the discovery set, already studied by WES and MiSeq targeted sequencing. We compared the patients' transcriptomes with those of 4 normal plasmacytoid dendritic cell (pDC) samples isolated from the peripheral blood of healthy individuals and used as controls. BPDCN tumor samples and $\mathrm{pDCs}$ segregated separately according to their gene expression profiles (Figure 2A). Two thousand and thirty-four genes (2034) were significantly deregulated among patients, and approximately half of them were up-regulated $(46 \%)$ in the BPDCN setting. Gene set enrichment analysis (GSEA) reported the significant deregulation of two genetic signatures involved in the methylation process, driven by the KDM5B34 histone demethylase and PRMT535 methyltransferase-associated genes, respectively. Of note, GSEA also detected the significant enrichment of a set of genes associated with the response to a DNA demethylating agent, ${ }^{36}$ namely decitabine (Figure 2B). The GSEA results [normalized enrichment score (NES) $\geq 2$; false discovery rate (FDR) qvalue $\leq 0.0001]$ were then validated in an extension set of 4 BPDCN samples and in a CAL-1 cell line (Online Supplementary Figures S4 and S5).

\section{Genome-wide ChIP-sequencing substantiates epigenetic dysregulation of cell cycle program in blastic plasmacytoid dendritic cell neoplasms}

To investigate if the transcriptional deregulation of BPDCNs could be linked to specific epigenetic features, we analyzed the histone acetylation/methylation profiles of 2 selected BPDCN patients (BPDCN_25 and BPDCN_37). The trimethylation at lysine 27 of histone 3 (H3K27me3) is closely associated with inactive gene promoters, while its acetylation (H3K27ac) closely correlates with gene activation, the two epigenetic modifications being mutually exclusive. Given this, we analyzed the genome-wide distribution of trimethylation and acetylation profiles of H3K27 in BPDCN cases. The analysis of PAT-ChIP sequencing data demonstrated that the 2 patients converged on the same pattern of histone acetylation, sharing as much as $43.6 \%$ of the acetylated promoters (Figure $2 \mathrm{C}$ and D). PAT-ChIP sequencing results were then integrated into the RNA sequencing data leading to the identification of a signature of 86 genes marked by promoter acetylation and significantly overexpressed in the BPDCN RNA sequencing sets. Gene 
Ontology analysis of the 86 selected genes highlighted the enrichment in biological processes involved in cell cycle progression (FDR q-value $<0.001$ ) (Figure $2 \mathrm{E}$ and Online Supplementary Table S11).

\section{In vivo blastic plasmacytoid dendritic cell neoplasm modeling demonstrates combined epigenetic therapy as effective in controlling disease progression}

The integration of results obtained from WES, RNA sequencing and PAT-ChIP-sequencing experiments clearly pointed to a fundamental role for epigenetic dysregulation in BPDCN and allowed us to hypothesize that this malignancy could display susceptibility to drugs active on the epigenetic regulation. Following the demonstration that the CAL-1 cell line, like primary BPDCN samples, had mutations clustering in chromatin remodeling pathway (Figure 1C) and enrichment in the same epigenetic programs (Online Supplementary Figure S5), we developed an in vivo CAL-1 xenograft BPDCN-like model to explore the effects of treatments targeting the acetylation, methylation, and also the NF-אB pathways, according to previous results. ${ }^{17,18}$ To this end, we focused on four

different

FDA-approved compounds: 5'-azacytidine, decitabine, romidepsin and bortezomib.

NSG mice intravenously injected with $5 \times 10^{3}$ CAL-1 cells rapidly developed a systemic BPDCN-like progressive disease, which was defined by the flow cytometry identification of human $\mathrm{CD} 56^{+} \mathrm{CD} 38^{+}$malignant cells in the peripheral blood, bone marrow, spleen and liver, as evaluated at 39 days after injection (Figure 3A). The pathological infiltration by malignant BPDCN cells in the mouse model was also confirmed at the same time point by the histopathological analysis of the bone marrow and spleen samples, which showed the presence of atypical cells with blastic morphology and expressing the human CD303/BDCA2 pDC marker (Figure 3B). Xenografted mice were divided into 11 treatment groups ( $\mathrm{n}=110$ mice) one day after CAL-1 injection and treated with either saline or with the hypo-methylating agents 5'-azacitidine or decitabine, the proteasome inhibitor bortezomib, and the histone deacetylase inhibitor romidepsin, used as single agents or in combination, according to the treatment schedule summarized in Figure 3C. The administration of 5 'azacytidine and decitabine used as single agents significantly prolonged OS of the mice when compared with saline (median survival 43.6 days vs. 32 days, $P<0.01$ for 5 '-azacytidine; median survival 44.7 days vs. 32 days, $P<0.05$ for decitabine) while neither bortezomib nor romidepsin alone showed beneficial effects on disease outcome. When the same agents were associated in combined treatment experiments, three different combinations were seen to significantly prolong mouse survival: i) the association of romidepsin and decitabine (median survival 42.8 days vs. 32 days, $P<0.05)$; ii) the combination of romidepsin, decitabine, and 5'-azacytidine (median survival 41.8 days vs. 32 days, $P<0.01$ ); and iii) the association of decitabine and 5'-azacytidine (median survival 52.8 days vs. 32 days, $P<0.01$ ), which achieved the best result in terms of survival (Figure 3D). Consistently, $5^{\prime}$ azacytdine and decitabine administered alone reduced the CAL-1-induced splenomegaly as evaluated at day 39 post injection and their combination proved to be even more effective (Figure 3E).

\section{Discussion}

Blastic plasmacytoid dendritic cell neoplasm is a rare myeloid malignancy with dismal prognosis and no standard therapy. In the present study, we performed WES on the largest series of BPDCNs that, to the best of our knowledge, has so far been reported in the literature. Thanks to the integration of WES with RNA and PATChIP sequencing, we provide new insights into BPDCN pathobiology by highlighting the dysregulation of the epigenetic program as a hallmark of the disease and suggest possible novel therapeutic interventions.

We found BPDCN patients extensively affected by mutations of genes involved in the epigenetic regulation: 25 epigenetic modifiers were mutated in almost all BPDCN patients (13 out of 14) and the CAL-1 cell line. In more than half of the patients (8 out of 14), the mutations heralded damaging functional alterations (Figure 1C). Some of the mutated genes have already been reported in previous studies (e.g. ASXL1, RAS, ATM, ARID1A, and $I D H 2)$, although, at times, at different rates than in our series (see ASXL1 and TET2, which were found to be mutated in $28.6 \%$ and $21.4 \%$ of our samples $v$ s. $32 \%$ and $36 \%$ of those of Menezes et al. ${ }^{19}$ ). In this respect, it should be remembered that the aim of our study was not only to extensively explore the mutational landscape of BPDCN, but also to possibly translate molecular notions into a preclinical approach. In any case, thanks to the employment of a WES approach, which did not limit our investigation to a priori-selected genes, we recognized additional mutated epigenetic factors that have never been described before but which are potentially relevant in the context of BPDCN, like PHF2 histone demethylase, that enhances the TP53-tumor suppressor activity, ${ }^{37}$ and the CHD 8 Chromodomain helicase DNA-binding protein-8, that promotes the E2F-dependent transcription and cell cycle progression. ${ }^{38}$ Besides the epigenetic pathway, we also detected mutations affecting programs common to other myeloid malignancies, such as DNA repair process, ${ }^{30}$ Wnt $/ \beta$-catenin signaling, ${ }^{31}$ and the differentiation pathway. ${ }^{32}$

Importantly, the functional enrichment analysis of WES data showed that among all genes/pathways explored the epigenetic program was the most deregulated $(P<0.0001)$.

To evaluate the impact of the identified epigenetic mutations at gene expression level, we analyzed the transcriptome of samples studied by WES. Among up-regulated genes, GSEA revealed the significant enrichment of two methylation pathways, driven respectively by the KDM5B histone-demethylase ${ }^{34}$ and by the PRMT5 arginine methyltransferase $-5 ;^{35}$ these two epigenetic modifiers are reported to be over-expressed in several cancer types and also represent promising therapeutic targets. ${ }^{39}$ Blockade of the PRMT5 activity reduces cell survival in chronic myelogenous leukemia ${ }^{40}$ and inhibition of $K D M 5 B$ demethylation correlates with cell growth arrest in hepatocellular carcinoma and breast cancers. ${ }^{41,42}$ We also identified the overexpression of one gene signature ${ }^{36}$ specifically responsive to the administration of the hypomethylating agent decitabine; a molecular finding bearing important therapeutic implications (FDR $q=1.85 \mathrm{E}-$ 5). To gain a functional insight into the epigenetic landscape of BPDCN samples, we performed PAT-ChIP sequencing of H3K27-acetylation/trymethylation signals of 2 BPDCN patients. The trimethylation of $\mathrm{H} 3 \mathrm{~K} 27$ marks 
inactive gene promoters and enhancers, while its acetylation correlates with gene activation. ${ }^{43}$ PAT-ChIP sequencing data showed that the 2 patients converged on the same epigenetic pattern sharing approximately half of the identified H3K27-acetylated promoters. Interestingly, the common acetylated regions comprised 10 superenhancers (SE) bound by the Bromodomain-containing protein 4 (BRD4), as described by Ceribelli et al. in a recent work on BPDCN (data not shown). ${ }^{24}$

The integration of PAT-ChIP and the RNA sequencing data highlighted a set of 86 genes involved in the cell-cycle progression aberrantly over-expressed and marked by H3K27-promoter acetylation. This finding suggests that the cell-cycle deregulation could be driven by H3K27acetylation signals, a hypothesis meriting future ad hoc studies that could help to clarify the mechanism of proliferation of this largely obscure disease.

The rarity of the disease (with an incidence of $0.000045 \%$ ) and its extremely aggressive behavior (OS 1019 months) limits the number of available patients included in biological and/or clinical studies. For these reasons, not surprisingly, BPDCN is still an orphan tumor lacking a standardized and effective therapeutic approach. In the last few years, new molecular studies have opened the way to innovative target therapies (e.g. bortezomib, ${ }^{17,18}$ venetoclax, ${ }^{22}$ BET-inhibitors, ${ }^{24}$ SL-40125) being used in clinical trials. Some of these are showing promising results, although still concerns remain regarding their safety. Of note, all the treatments proposed are mainly the result of investigation into the RNA transcriptome, while the DNA features of BPDCN patients have barely been evaluated.

We therefore decided to tackle this yet incurable disease by designing the first therapeutic strategy modeled on the DNA mutational status of BPDCN patients, analyzed by WES. The WES mutational findings enhanced by the RNA and PAT-ChIP sequencing results clearly evidenced the prominent role of the epigenetic program dysregulation among $\mathrm{BPDCN}$ patients and guided our therapeutic approach towards the use of epigenetic agents. In particular, we tested in vivo the efficacy of US Food and Drug Administration-approved epigenetic drugs which could be considered for potential repositioning in clinical trials: two hypomethylating agents such as decitabine and 5'-azacytidine, and the histone deacetylase inhibitor romidepsin. We hypothesized that these drugs could impact on tumor progression because: i) BPDCN patients displayed potential sensitivity to hypomethylating agents, particularly to decitabine, as detected by GSEA analysis; ii) both 5'-azacytidine and decitabine are currently used for the treatment of myelodysplastic syndromes, ${ }^{44,45}$ which are myeloid neoplasms sharing many epigenetic mutated genes with BPDCN; iii) preclinical studies on several malignancies demonstrated that the action of decitabine is synergized by romidepsin. ${ }^{46}$ In the light of this, our experimental design focused on epigenetic drugs with a largescale activity, aiming to explore whether we might induce cell death by perturbation of the malignant epigenetic programme. In addition to the epigenetic drugs, we also verified the efficacy of bortezomib, a proteasome inhibitor, which had previously been shown to significantly induce in vitro and in vivo BPDCN cell death. ${ }^{17,18}$ Our experiments revealed that the treatment with 5'-azacytidine in combination with decitabine significantly inhibits disease progression and extends survival $(P<0.01)$ in a preclinical mouse model. In the past, two reports experimented the use of 5'-azacytidine in elderly BPDCN patients, though this therapeutic choice was not yet sustained by a molecular rationale. ${ }^{47,48}$ Here we demonstrate that 5 '-azacytidine is more effective in tumor eradication when combined with decitabine. Further studies are ongoing to elucidate the synergistic mechanisms between the two drugs.

In conclusion, we have identified the deregulation of the epigenetic program as a genetic hallmark of BPDCN and suggest a novel therapeutic approach based on the combination of two hypomethylating agents, 5'-azacytidine and decitabine, to be tested in future clinical trials.

\section{Funding}

The present work was supported by the AIRC grants IG 15762 and $5 \times 100010007$ "Genetics-driven targeted management of lymphoid malignancies" and the Grant "Innovative approaches to the diagnosis and pharmacogenetic-based therapies of primary hepatic tumours, peripheral B and T-cell lymphomas and lymphoblastic leukaemias" Strategic Programme 2010-2012 Regione Emilia Romagna - Università (all to SAP).

\section{References}

1. Chaperot L, Bendriss N, Manches $O$, et al. Identification of a leukemic counterpart of the plasmacytoid dendritic cells. Blood. 2001;97(10):3210-3217

2. Swerdlow SH, Campo E, Hazzis NL, et al. Facchetti F, Jones D, Petrella T. Blastic plasmacytoid dendritic cell neoplasm. In: Swerdlow SH, Campo E, Hazzis NL, et al., eds. WHO Classification of Tumors of Haematopoietic and Lymphoid Tissues. Lyon: IARC Press; 2008:145-147.

3. Facchetti F, Cigognetti M, Fisogni S, Rossi G, Lonardi S, Vermi W. Neoplasms derived from plasmacytoid dendritic cells. Mod Pathol. 2016;29(2):98-111.

4. Garnache-Ottou F, Feuillard J, Ferrand C, et al. Extended diagnostic criteria for plasmacytoid dendritic cell leukaemia. $\mathrm{Br} J$ Haematol. 2009;145(5):624-636.

5. Pagano L, Valentini CG, Pulsoni A, et al.
Blastic plasmacytoid dendritic cell neoplasm with leukemic presentation: an Italian multicenter study. Haematologica. 2013;98(2): 239-246.

6. Roos-Weil D, Dietrich S, Boumendil A, et al. Stem cell transplantation can provide durable disease control in blastic plasmacytoid dendritic cell neoplasm: a retrospective study from the European Group for Blood and Marrow Transplantation. Blood. 2013;121(3):440-446.

7. Pemmaraju N. Blastic plasmacytoid dendritic cell neoplasm. Clin Adv Hematol Oncol. 2016;14(4):220-222.

8. Petrella T, Dalac S, Maynadie M, et al. CD4+ CD56+ cutaneous neoplasms: a distinct hematological entity? Groupe Francais d'Etude des Lymphomes Cutanes (GFELC). Am J Surg Pathol. 1999;23(2):137-146.

9. Leroux D, Mugneret F, Callanan $\mathrm{M}$, et al. CD4(+), CD56(+) DC2 acute leukemia is characterized by recurrent clonal chromosomal changes affecting 6 major targets: a study of 21 cases by the Groupe Francais de Cytogenetique Hematologique. Blood. 2002;99(11):4154-4159.

10. Reichard KK, Burks EJ, Foucar MK, et al. CD4(+) CD56(+) lineage-negative malignancies are rare tumors of plasmacytoid dendritic cells. Am J Surg Pathol. 2005;29(10):12741283.

11. Dijkman R, van Doorn R, Szuhai K, Willemze R, Vermeer MH, Tensen CP. Gene-expression profiling and array-based CGH classify CD4+CD56+ hematodermic neoplasm and cutaneous myelomonocytic leukemia as distinct disease entities. Blood. 2007;109(4):1720-1727.

12. Wiesner T, Obenauf AC, Cota C, Fried I, Speicher MR, Cerroni L. Alterations of the cell-cycle inhibitors p27(KIP1) and p16(INK4a) are frequent in blastic plasmacytoid dendritic cell neoplasms. J Invest Dermatol. 2010;130(4):1152-1157.

13. Agliano A, Martin-Padura I, Marighetti P, et al. Therapeutic effect of lenalidomide in a 
novel xenograft mouse model of human blastic NK cell lymphoma/blastic plasmacytoid dendritic cell neoplasm. Clin Cancer Res. 2011:17(19):6163-6173.

14. Jardin F, Ruminy P, Parmentier F, et al. TET2 and TP53 mutations are frequently observed in blastic plasmacytoid dendritic cell neoplasm. Br J Haematol. 2011;153(3):413-416.

15. Lucioni M, Novara F, Fiandrino G, et al. Twenty-one cases of blastic plasmacytoid dendritic cell neoplasm: focus on biallelic locus 9p21.3 deletion. Blood. 2011;118(17):4591-4594.

16. Alayed K, Patel KP, Konoplev S, et al. TET2 mutations, myelodysplastic features, and a distinct immunoprofile characterize blastic plasmacytoid dendritic cell neoplasm in the bone marrow. Am J Hematol. 2013;88(12):1055-1061.

17. Sapienza MR, Fuligni F, Agostinelli C, et al. Molecular profiling of blastic plasmacytoid dendritic cell neoplasm reveals a unique pattern and suggests selective sensitivity to NF$\mathrm{kB}$ pathway inhibition. Leukemia. 2014:28(8):1606-1616.

18. Philippe L, Ceroi A, Bole-Richard E, et al. Bortezomib as a new therapeutic approach for blastic plasmacytoid dendritic cell neoplasm. Haematologica. 2017;102(11):18611868.

19. Menezes J, Acquadro F, Wiseman M, et al. Exome sequencing reveals novel and recurrent mutations with clinical impact in blastic plasmacytoid dendritic cell neoplasm. Leukemia. 2014;28(4):823-829.

20. Stenzinger A, Endris V, Pfarr N, et al. Targeted ultra-deep sequencing reveals recurrent and mutually exclusive mutations of cancer genes in blastic plasmacytoid dendritic cell neoplasm. Oncotarget. 2014;5(15): 6404-6413.

21. Emadali A, Hoghoughi N, Duley S, et al. Haploinsufficiency for NR3C1, the gene encoding the glucocorticoid receptor, in blastic plasmacytoid dendritic cell neoplasms. Blood. 2016;127(24):3040-3053.

22. Montero J, Stephansky J, Cai T, et al. Blastic Plasmacytoid Dendritic Cell Neoplasm Is Dependent on BCL2 and Sensitive to Venetoclax. Cancer Discov. 2017;7(2):156164

23. Ceroi A, Masson D, Roggy A, et al. LXR agonist treatment of blastic plasmacytoid dendritic cell neoplasm restores cholesterol efflux and triggers apoptosis. Blood. 2016;128(23):2694-2707.

24. Ceribelli M, Hou ZE, Kelly PN, et al. A Druggable TCF4- and BRD4-Dependent Transcriptional Network Sustains Malignancy in Blastic Plasmacytoid
Dendritic Cell Neoplasm. Cancer Cell. 2016;30(5):764-778.

25. Angelot-Delettre F, Roggy A, Frankel AE, et al. In vivo and in vitro sensitivity of blastic plasmacytoid dendritic cell neoplasm to SL401, an interleukin-3 receptor targeted biologic agent. Haematologica. 2015;100(2): 223-230.

26. Fanelli M, Amatori S, Barozzi I, et al. Pathology tissue-chromatin immunoprecipitation, coupled with high-throughput sequencing, allows the epigenetic profiling of patient samples. Proc Natl Acad Sci U S A. 2010;107(50):21535-21540.

27. Maeda T, Murata K, Fukushima T, et al. A novel plasmacytoid dendritic cell line, CAL1 , established from a patient with blastic natural killer cell lymphoma. Int J Hematol. 2005;81(2):148-154

28. Ashburner M, Ball CA, Blake JA, et al. Gene ontology: tool for the unification of biology. The Gene Ontology Consortium. Nat Genet. 2000;25(1):25-29.

29. Ward AF, Braun BS, Shannon KM. Targeting oncogenic Ras signaling in hematologic malignancies. Blood. 2012; 120(17):3397 3406.

30. Guarini A, Marinelli M, Tavolaro S, et al. ATM gene alterations in chronic lymphocytic leukemia patients induce a distinct gene expression profile and predict disease progression. Haematologica. 2012;97(1):47-55.

31. Simon M, Grandage VL, Linch DC, Khwaja A. Constitutive activation of the Wnt/betacatenin signalling pathway in acute myeloid leukaemia. Oncogene. 2005; 24(14):2410 2420.

32. Kuo YH, Zaidi SK, Gornostaeva S, Komori T, Stein GS, Castilla LH. Runx2 induces acute myeloid leukemia in cooperation with Cbfbeta-SMMHC in mice. Blood. 2009;113(14):3323-3332.

33. Togami K, Madan V, Li J, et al. Blastic Plasmacytoid Dendritic Cell Neoplasm (BPDCN) Harbors Frequent Splicesosome Mutations That Cause Aberrant RNA Splicing Affecting Genes Critical in pDC Differentiation and Function. Blood. 2016; 128(22):738.

34. Scibetta AG, Santangelo S, Coleman J, et al. Functional analysis of the transcription repressor PLU-1/JARID1B. Mol Cell Biol. 2007;27(20):7220-7235

35. Pal S, Vishwanath SN, Erdjument-Bromage H, Tempst P, Sif S. Human SWI/SNF-associted PRMT5 methylates histone $\mathrm{H} 3$ arginine 8 and negatively regulates expression of ST7 and NM23 tumor suppressor genes. Mol Cell Biol. 2004;24(21):9630-9645.

36. Missiaglia E, Donadelli M, Palmieri M,
Crnogorac-Jurcevic T, Scarpa A, Lemoine NR. Growth delay of human pancreatic cancer cells by methylase inhibitor 5-aza-2' deoxycytidine treatment is associated with activation of the interferon signalling pathway. Oncogene. 2005;24(1):199-211

37. Lee KH, Park JW, Sung HS, et al. PHF2 histone demethylase acts as a tumor suppressor in association with p53 in cancer. Oncogene. 2015;34(22):2897-2909.

38. Subtil-Rodriguez A, Vazquez-Chavez E Ceballos-Chavez $\mathrm{M}$, et al. The chromatin remodeller CHD8 is required for E2F-dependent transcription activation of S-phase genes. Nucleic Acids Res. 2014;42(4):2185 2196.

39. Rotili D, Mai A. Targeting Histone Demethylases: A New Avenue for the Fight against Cancer. Genes Cancer. 2011;2(6): 663-679.

40. Jin Y, Zhou J, Xu F, et al. Targeting methyl transferase PRMT5 eliminates leukemia stem cells in chronic myelogenous leukemia. J Clin Invest. 2016;126(10):3961-3980.

41. Yamane K, Tateishi K, Klose RJ, et al. PLU-1 is an H3K4 demethylase involved in transcriptional repression and breast cancer cell proliferation. Mol Cell. 2007;25(6):801-812.

42. Tang B, Oi G, Tang F, et al. JARID1B promotes metastasis and epithelial-mesenchy mal transition via PTEN/AKT signaling in hepatocellular carcinoma cells. Oncotarget. 2015;6(14):12723-12739

43. Zhang T, Cooper S, Brockdorff N. The interplay of histone modifications - writers that read. EMBO Rep. 2015;16(11):1467-1481.

44. Quintas-Cardama A, Santos FP, GarciaManero G. Therapy with azanucleosides for myelodysplastic syndromes. Nat Rev Clin Oncol. 2010;7(8):433-444.

45. Jabbour E, Short NJ, Montalban-Bravo G, et al. Randomized phase 2 study of low-dose decitabine vs low-dose azacitidine in lowerrisk MDS and MDS/MPN. Blood. 2017;130(13):1514-1522

46. Kalac M, Scotto L, Marchi E, et al. HDAC inhibitors and decitabine are highly synergistic and associated with unique geneexpression and epigenetic profiles in models of DLBCL. Blood. 2011;118(20):55065516

47. Laribi K, Denizon N, Ghnaya H, et al. Blastic plasmacytoid dendritic cell neoplasm: the first report of two cases treated by 5-azacy tidine. Eur J Haematol. 2014;93(1):81-85.

48. Khwaja R, Daly A, Wong M, Mahe E, Cerquozzi S, Owen C. Azacitidine in the treatment of blastic plasmacytoid dendritic cell neoplasm: a report of 3 cases. Leuk Lymphoma. 2016;57(11):2720-2722. 